\title{
Gruppi fattorizzati da sottogruppi ciclici
}

\author{
ENRICO JABARA (*)
}

Al professor Federico Menegazzo per il suo $65^{\circ}$ compleanno

ABstRaCT - This paper is devoted to a study of groups defined by the presentation

$$
G=\left\langle a, b, c \mid a^{b}=a^{1+r}, b^{c}=b^{1+s}, c^{a}=c^{1+t}\right\rangle \quad(r, s, t \in \mathbb{Z}) .
$$

It is proved that $G^{\prime \prime} \leq Z(G)$ and that if $r, s$ and $t$ are all $\neq-2,0$ then $G$ is finite and its order divides $|(r, s)(s, t)(t, r) \rho \sigma \tau|$ where $\rho=(1+r)^{|s|}-1$, $\sigma=(1+s)^{|t|}-1$ and $\tau=(1+t)^{|r|}-1$.

\section{Introduzione.}

Lo scopo che questo lavoro si prefigge è duplice. In primo luogo si continua lo studio, iniziato in [6], dei gruppi fattorizzati tramite tre (o più) sottogruppi abeliani. In secondo luogo si applicano alcuni dei risultati ottenuti allo studio della famiglia di gruppi definiti dalla seguente presentazione:

$$
M(r, s, t)=\left\langle a, b, c \mid a^{b}=a^{1+r}, b^{c}=b^{1+s}, c^{a}=c^{1+t}\right\rangle ; \quad r, s, t \in Z .
$$

In [9] Mennicke ha studiato i gruppi $M(t, t, t)$ ed ha dimostrato che se $t \geq 1$ si tratta di gruppi finiti in cui il sottogruppo $\left\langle a^{t^{3}}, b^{t^{3}}, c^{t^{3}}\right\rangle$ è normale, abeliano e a quoziente nilpotente. Successivamente Schenkman in [13] ha dimostrato che il secondo derivato di $M(r, s, t)$ è nilpotente di classe al più 3 e che $M(r, s, t)$ è finito se $r, s, t$ sono tutti maggiori di 0 . In questo lavoro si dimostra il

(*) Indirizzo dell'A.: Dipartimento di Matematica Applicata, Università di Ca' Foscari, Dorsoduro 3825/e, 30123 Venezia, Italy.

E-mail: jabara@unive.it

2000 Mathematical Subject Classification: 20D40 (20F05,17B60). 
TeORema 1. Perogni $r, s, t \in Z$ il gruppo $G=M(r, s, t) \grave{e}$ supersolubile e si ha $G^{\prime \prime} \leq Z(G)$ e $\gamma_{3}(G) \leq Z\left(G^{\prime}\right)$. In particolare $G^{\prime}$ è nilpotente di classe al più 2. Inoltre se $r, s, t$ sono tutti diversi da 0 e da -2 allora $G$ è finito e il suo ordine divide

$$
|(r, s)(s, t)(t, r) \rho \sigma \tau|
$$

ove $\rho=(1+r)^{|s|}-1, \sigma=(1+s)^{|t|}-1, \tau=(1+t)^{|r|}-1 e(m, n)$ indica il massimo comun divisore tra $i$ due numeri interi $m$ e $n$.

La dimostrazione del Teorema 1 è ottenuta combinando alcuni risultati più generali riguardanti i gruppi fattorizzati con dei calcoli diretti sui commutatori. Si dimostrerà anche la seguente generalizzazione del Teorema 3 di [13].

Proposizione 1. Sia $G$ un gruppo generato da tre suoi sottogruppi $A$, $B$ e $C$ nilpotenti di classi rispettivamente $k_{A}, k_{B}$ e $k_{C}$. Se $[A, B] \leq A$, $[B, C] \leq B$ e $[C, A] \leq C$ allora, posto $K=k_{A}+k_{B}+k_{C}$, si ha che il gruppo $\gamma_{K+1}(G)$ risulta nilpotente di classe al più $K$.

Dalla Proposizione 1 discende che se $A, B$ e $C$ sono sottogruppi abeliani di $G$ allora $\gamma_{4}\left(\gamma_{4}(G)\right)=\{1\}$; sotto tali ipotesi si può ottenere un risultato più preciso.

Proposizione 2. Sia $G$ un gruppo generato da tre suoi sottogruppi abeliani $A, B$ e $C$. Se $[A, B] \leq A,[B, C] \leq B$ e $[C, A] \leq C$ allora $G^{\prime}$ risulta nilpotente di classe al più 3 .

È conveniente riformulare la prima parte del Teorema 1.

Proposizione 3. Sia $G$ un gruppo generato da tre suoi sottogruppi ciclici $A, B$ e $C$. Se $[A, B] \leq A,[B, C] \leq B$ e $[C, A] \leq C$ allora $G$ risulta supersolubile e si ha $G^{\prime \prime} \leq Z(G)$ e $\gamma_{3}(G) \leq Z\left(G^{\prime}\right)$. In particolare $G^{\prime}$ risulta nilpotente di classe al più 2 .

Le Proposizioni 1, 2 e 3 non si possono estendere al caso di quattro o più sottogruppi; infatti Higman in [4] ha dimostrato che il gruppo

$$
\left\langle a, b, c, d \mid a^{b}=a^{2}, b^{c}=b^{2}, c^{d}=c^{2}, d^{a}=d^{2}\right\rangle
$$

è infinito e privo di sottogruppi di indice finito.

Nel §3 sarà dimostrato un analogo del Teorema 1 valido per gli anelli di Lie. 


\section{Dimostrazione delle Proposizioni 1 e 2.}

Dimostrazione della Proposizone 1. Sia $G=\langle A, B, C\rangle \operatorname{con} A, B$ e $C$ soddisfacenti alle ipotesi dell'enunciato. Essendo $A B=B A, A C=C A$ e $B C=C B$ si deve avere $G=A B C$. Si ha poi $A^{G}=A^{A B C}=A^{C} \leq A C$ e, analogamente, $B^{G} \leq A B$ e $C^{G} \leq B C$. Poiché $C$ è normalizzato da $A$ e $\gamma_{k_{A}+1}(A)=\{1\}$ risulta $\gamma_{k_{A}+1}\left(A^{G}\right) \leq \gamma_{k_{A}+1}(A C) \leq C$. Siccome $A^{G} \unlhd G$ e $\gamma_{k_{A}+1}\left(A^{G}\right)$ è caratteristico in $A^{G}$ si ha $\gamma_{k_{A}+1}\left(A^{G}\right) \unlhd G$ e quindi $\gamma_{k_{A}+1}\left(A^{G}\right) \leq C_{G}$. In maniera analoga si dimostra che $\gamma_{k_{B}+1}\left(B^{G}\right) \leq A_{G}$ e $\gamma_{k_{C}+1}\left(C^{G}\right) \leq B_{G}$.

I tre sottogruppi $A_{G}, B_{G}$ e $C_{G}$ sono normali in $G$ e nilpotenti di classe che non supera rispettivamente $k_{A}, k_{B}$ e $k_{C}$. Quindi il sottogruppo $L=A_{G} B_{G} C_{G}$ è normale in $G$ e, per il teorema di Fitting (5.2.8 di [12]), nilpotente di classe al più $k_{A}+k_{B}+k_{C}=K$. Posto $\bar{G}=G / L$ in $\bar{G}$ si ha

$$
\gamma_{k_{A}+1}\left(\bar{A}^{\bar{G}}\right)=\{1\}, \quad \gamma_{k_{B}+1}\left(\bar{B}^{\bar{G}}\right)=\{1\}, \quad \gamma_{k_{C}+1}\left(\bar{C}^{\bar{G}}\right)=\{1\}
$$

e poiché $\bar{G}=\bar{A} \bar{B} \bar{C}=\bar{A}^{\bar{G}} \bar{B} \bar{G} \bar{C}^{\bar{G}}$, ancora per il teorema di Fitting, si conclude che $\bar{G}$ è nilpotente e che la sua classe di nilpotenza non supera $k_{A}+k_{B}+k_{C}=K$. Dunque $\gamma_{K+1}\left(\gamma_{K+1}(G)\right)=\{1\}$.

Per dimostrare la Proposizione 2 si utilizza il seguente risultato.

Lemma 1. Sia $G$ un gruppo e $A, B$ e $C$ dei sottogruppi abeliani di $G$ tali che $G=A B C,[A, B] \leq A,[B, C] \leq B,[C, A] \leq C$ e $A \cap B \cap C=\{1\}$. Allora $\left[G^{\prime}, G^{\prime}, G^{\prime}\right] \leq Z(G)$.

DIM. Sotto le ipotesi dell'enunciato risulta $A_{G} \cap B_{G} \leq Z(G)$; infatti essendo $A$ e $B$ abeliani essi sono centralizzati da $A_{G} \cap B_{G}$. Sia poi $x \in A_{G} \cap B_{G}$ e $y \in C$; poiché $A$ normalizza $C$ si ha $[x, y] \in C$, del resto $A_{G} \cap B_{G} \unlhd G$ porge che $[x, y] \in A_{G} \cap B_{G}$ e allora $[x, y] \in A_{G} \cap B_{G} \cap C=\{1\}$. Quindi $A_{G} \cap B_{G}$ centralizza anche $C$ e dunque $A_{G} \cap B_{G} \leq Z(G)$.

Ragionando come nella dimostrazione precedente e ricordando che $A, B$ e $C$ sono abeliani, si ottiene $\left(A^{G}\right)^{\prime} \leq C_{G},\left(B^{G}\right)^{\prime} \leq A_{G}$ e $\left(C^{G}\right)^{\prime} \leq B_{G}$.

Per dimostrare l'asserto si distinguono tre casi.

(a) Almeno due dei tre sottogruppi $A_{G}, B_{G}$ e $C_{G}$ risultano identici. Non è restrittivo supporre $A_{G}=\{1\}$ e $B_{G}=\{1\}$. Allora $\left(B^{G}\right)^{\prime}=\{1\}$ e $\left(C^{G}\right)^{\prime}=\{1\}$ e quindi il sottogruppo normale $N=B^{G} C^{G}$ risulta, per il teorema di Fitting, nilpotente di classe al più 2 . Siccome $G / N$ è isomorfo a un quoziente di $A$, che è 
abeliano, si ha $G^{\prime} \leq N$, da cui $\left[G^{\prime}, G^{\prime}, G^{\prime}\right]=\{1\}$ e in questo caso l'asserto è dimostrato.

(b) Uno solo dei tre sottogruppi $A_{G}, B_{G}$ e $C_{G}$ risulta identico.

Non è restrittivo supporre che $C_{G}=\{1\}$ e dunque $A^{G}$ è abeliano. In $\bar{G}=G / A_{G}$ anche $\bar{B} \bar{G}$ è abeliano e quindi, ragionando come nel punto precedente, si ricava che $\left(G / A_{G}\right)^{\prime}$ è nilpotente di classe al più 2 così come $\left(G / B_{G}\right)^{\prime}$. Ma allora $\left[G^{\prime}, G^{\prime}, G^{\prime}\right] \leq$ $\leq A_{G} \cap B_{G} \leq Z(G)$ e l'asserto è dimostrato.

(c) $A_{G} \neq\{1\}, B_{G} \neq\{1\}$ e $C_{G} \neq\{1\}$.

Allora siccome $A_{G} \cap B_{G} \cap C_{G} \leq A \cap B \cap C=\{1\}$, il gruppo $G$ si immerge nel prodotto diretto $\left(G / A_{G}\right) \times\left(G / B_{G}\right) \times\left(G / C_{G}\right)$ e poiché ognuno dei tre fattori del prodotto diretto ricade nel caso considerato nel punto (b) se ne conclude che $\left[G^{\prime}, G^{\prime}, G^{\prime}\right] \leq Z(G)$.

Quindi in ogni caso $\left[G^{\prime}, G^{\prime}, G^{\prime}\right] \leq Z(G)$ e l'asserto è dimostrato.

A questo punto la dimostrazione della Proposizione 2 è quasi immediata.

Dimostrazione della Proposizione 2. Poiché $A, B$ e $C$ sono abeliani, risulta $A \cap B \cap C \leq Z(G)$. In $\widehat{G}=G /(A \cap B \cap C)$ si ha $\widehat{A} \cap \widehat{B} \cap \widehat{C}=\{1\} \mathrm{e}$ quindi, per il Lemma 1 , $\left[\widehat{G}^{\prime}, \widehat{G}^{\prime}, \widehat{G}^{\prime}\right] \leq Z(\widehat{G})$ da cui $\left[G^{\prime}, G^{\prime}, G^{\prime}\right] \leq Z_{2}(G)$. In ogni gruppo $X$ si ha $\left[X^{\prime}, Z_{2}(X)\right]=\{1\}$, quindi $\left[G^{\prime}, G^{\prime}, G^{\prime}, G^{\prime}\right] \leq$ $\leq\left[Z_{2}(G), G^{\prime}\right]=\{1\}$ e $G^{\prime}$ risulta nilpotente di classe al più 3 .

Osservazione 1. Con gli stessi medodi utilizzati nella dimostrazione della Proposizione 1 si può far vedere che se $G=\langle A, B, C\rangle$ con $A, B$ e $C$ risolubili di lunghezza derivata rispettivamente $d_{A}, d_{B}$ e $d_{C}$ e tali che $[A, B] \leq A,[B, C] \leq B$ e $[C, A] \leq C$, allora anche $G$ è risolubile e la sua lunghezza derivata non supera $2\left(d_{A}+d_{B}+d_{C}\right)$. Inoltre, poiché $G=A B C$, se $A, B$ e $C$ sono policiclici, anche $G$ risulta policiclico.

OSSERVAZIONE 2. Se $G$ è un gruppo finito generato da tre sottogruppi ciclici $A, B$ e $C$ tali che $[A, B] \leq A,[B, C] \leq B$ e $[C, A] \leq C$, allora, ragionando come nella dimostrazione del Lemma 1 e della Proposizione 2 (e sfruttando il fatto che ogni sottogruppo di $A$ è normalizzato da $B$, ogni sottogruppo di $B$ è normalizzato da $C$ e ogni sottogruppo di $C$ è normalizzato da $A$ ) si può dimostrare che $G^{\prime \prime} \leq Z_{2}(G)$ (e di conseguenza $G^{\prime}$ risulta nilpotente di classe al più 2). La dimostrazione che $G^{\prime \prime} \leq Z(G)$ richiede, come sarà chiaro nel $\S 4$, maggiore attenzione. 


\section{Anelli di Lie.}

Da una lettura delle dimostrazioni precedenti è facile convincersi che le Proposizioni 2 e 3 (in analogia a quanto avviene per il Teorema 2 di [13]) valgono anche per gli anelli di Lie (su Z). Più precisamente se $\mathcal{L}$ è un anello di Lie e $\mathfrak{A}, \mathfrak{B}$ e $\mathfrak{C}$ sono suoi sottanelli tali che $\mathfrak{Q}=\mathfrak{A}+\mathfrak{B}+\mathfrak{C}$ e $[\mathfrak{U}, \mathfrak{B}] \leq \mathfrak{A}$, $[\mathfrak{B}, \mathfrak{C}] \leq \mathfrak{B}$ e $[\mathfrak{C}, \mathfrak{H}] \leq \mathfrak{C}$ allora

(i) se $\mathfrak{A}, \mathfrak{B}$ e $\mathfrak{\complement}$ sono nilpotenti di classe rispettivamente $k_{\mathfrak{A}}, k_{\mathfrak{B}}$ e $k_{\mathfrak{C}}$ allora, detto $K=k_{\mathfrak{A}}+k_{\mathfrak{B}}+k_{\mathfrak{S}}$, si ha $\left(\mathfrak{Q}^{K+1}\right)^{K+1}=\{0\}$;

(ii) se $\mathfrak{A}, \mathfrak{B}$ e $\mathfrak{S}$ sono abeliani allora $\left[\mathfrak{R}^{\prime}, \mathfrak{R}^{\prime}, \mathfrak{R}^{\prime}\right] \leq Z_{2}(\mathfrak{R})$ e quindi, in particolare, $\mathbb{R}^{\prime}$ è nilpotente di classe al più 3 .

Ove, come d'uso si pone $\mathfrak{Q}^{1}=\mathfrak{Q}, \mathfrak{Q}^{n}=\left[\mathfrak{Q}^{n-1}, \mathfrak{Q}\right], \mathfrak{Q}^{\prime}=\mathfrak{Q}^{2}$ e $\mathfrak{Q}^{\prime \prime}=\left[\mathfrak{Q}^{\prime}, \mathfrak{Q}^{\prime}\right]$.

Si supponga ora che $\mathfrak{A}, \mathfrak{B}$ e $\mathfrak{C}$ siano generati, come anelli, da $\mathfrak{a}, \mathfrak{b}$ e $\mathfrak{c}$ rispettivamente. Se si ha $[\mathfrak{a}, \mathfrak{b}]=r \mathfrak{a},[\mathfrak{b}, \mathfrak{c}]=s \mathfrak{b},[\mathfrak{c}, \mathfrak{a}]=t \mathfrak{a}$ con $r, s, t \in \mathbb{Z}$, l'anello di Lie generato da $\mathfrak{a}, \mathfrak{b}$ e $\mathfrak{c}$ sarà denotato con $L(r, s, t)$. Utilizzando l'identatà di Jacobi $[\mathfrak{a}, \mathfrak{b}, \mathfrak{c}]+[\mathfrak{b}, \mathfrak{c}, \mathfrak{a}]+[\mathfrak{c}, \mathfrak{a}, \mathfrak{b}]=0$, si ricava

$$
r s \mathfrak{a}+s t \mathfrak{b}+r t \mathfrak{c}=0 .
$$

da cui (commutando rispetto $\mathfrak{a}, \mathfrak{b}$ e $\mathfrak{c}$ ) si ottiene

$$
r s t \mathfrak{a}=r t^{2} \mathfrak{c}, \quad r s t \mathfrak{b}=r^{2} s \mathfrak{a}, \quad r s t \mathfrak{c}=s^{2} t \mathfrak{b} .
$$

Commutando (2.b) tramite $\mathfrak{b}$ e $\mathfrak{a}$, (2.c) tramite $\mathfrak{c}$ e $\mathfrak{b}$ e (2.a) tramite $\mathfrak{a}$ e $\mathfrak{c}$, si ottiene

$$
\begin{array}{rlrl}
r^{3} s \mathfrak{a} & =0, & s^{3} t \mathfrak{b}=0, & r t^{3} \mathfrak{c}=0, \\
r^{2} s t \mathfrak{a}=0, & r s^{2} t \mathfrak{b}=0, & r s t^{2} \mathfrak{c}=0 .
\end{array}
$$

Grazie alle (4) si ricava immediatamente che se $r s t \neq 0$ allora $L(r, s, t)$ è finito e il suo ordine non supera $(r s t)^{4}$.

Moltiplicando le relazioni (2) rispettivamente per $t, r$ e $s$ si ricava

$$
r s t^{2} \mathfrak{a}=0, \quad r^{2} s t \mathfrak{b}=0, \quad r s^{2} t \mathfrak{c}=0 .
$$

Da (2) applicando le (4) si ottiene

$$
r s^{2} t \mathfrak{a}=0, \quad r s t^{2} \mathfrak{b}=0, \quad r^{2} s t \mathfrak{c}=0 .
$$

Moltiplicando (1) rispettivamente per $r s$, rt e $s r$ e tenendo conto delle (4) si ha anche

$$
r^{2} s^{2} \mathfrak{a}=0, \quad s^{2} t^{2} \mathfrak{b}=0, \quad r^{2} t^{2} \mathfrak{c}=0 .
$$

Si può quindi enunciare il 
TEOREMA 2. Se $\mathfrak{R}=L(r, s, t)$ allora si $h a$

(a) $\Omega^{\prime \prime} \leq Z(\Omega)$;

(b) $\left[\mathfrak{Q}^{3}, \mathfrak{R}^{2}\right]=\{0\}$.

Inoltre se rst $\neq 0$ allora $\mathfrak{Q}$ è finito e $i l$ suo ordine divide $\left|(r, s, t)^{3} r^{2} s^{2} t^{2}\right|$ ove $(r, s, t)$ denota il massimo comun divisore dei numeri interi $r$, s e $t$.

Dim. Siccome $\mathfrak{R}^{\prime}$ è generato da $r a, s \mathfrak{b}$ e $t c$ si ha che $\mathfrak{L}^{\prime \prime}$ è generato da $r^{2} s a$, $s^{2} t \mathfrak{b}$ e $r t^{2} \mathfrak{c}$. Sfruttando le uguaglianze (3) si ottiene

$$
\left[r^{2} s \mathfrak{a}, \mathfrak{b}\right]=r^{3} s \mathfrak{a}=0, \quad\left[s^{2} t \mathfrak{b}, \mathfrak{c}\right]=s^{3} t \mathfrak{b}=0, \quad\left[r t^{2} \mathfrak{c}, \mathfrak{a}\right]=r t^{3} \mathfrak{c}=0,
$$

mentre dalle uguaglianze (5) si ricava

$$
\left[r^{2} s \mathfrak{a}, \mathfrak{c}\right]=-r^{2} s t \mathfrak{c}=0, \quad\left[s^{2} t \mathfrak{b}, \mathfrak{a}\right]=-r s^{2} t \mathfrak{a}=0, \quad\left[r t^{2} \mathfrak{c}, \mathfrak{b}\right]=-r s t^{2} \mathfrak{b}=0,
$$

e l'asserto (a) è dimostrato.

Siccome $\mathfrak{2}^{3}$ è generato da $r^{2} \mathfrak{a}, s^{2} \mathfrak{b}, t^{2} \mathfrak{c}, r s a, s t \mathfrak{b}, r t \mathfrak{c}$, ricordando (3), (4) e (7) si ottiene:

$$
\begin{array}{lll}
{\left[r^{2} \mathfrak{a}, s \mathfrak{b}\right]=r^{3} s \mathfrak{a}=0,} & {\left[s^{2} \mathfrak{b}, t \mathfrak{c}\right]=s^{3} t \mathfrak{b}=0,} & {\left[t^{2} \mathfrak{c}, r \mathfrak{a}\right]=r t^{3} \mathfrak{c}=0,} \\
{\left[r^{2} \mathfrak{a}, t \mathfrak{c}\right]=-r^{2} t^{2} \mathfrak{c}=0,} & {\left[s^{2} \mathfrak{b}, r \mathfrak{a}\right]=-r^{2} s^{2} \mathfrak{a}=0,} & {\left[t^{2} \mathfrak{c}, s \mathfrak{b}\right]=-s^{2} t^{2} \mathfrak{b}=0,} \\
{[r s \mathfrak{a}, s \mathfrak{b}]=r^{2} s^{2} \mathfrak{a}=0,} & {[s t \mathfrak{b}, t \mathfrak{c}]=s^{2} t^{2} \mathfrak{b}=0,} & {[r t \mathfrak{c}, r \mathfrak{a}]=r^{2} t^{2} \mathfrak{c}=0,} \\
{[r s \mathfrak{a}, t \mathfrak{c}]=-r s t^{2} \mathfrak{c}=0,} & {[s t \mathfrak{b}, r \mathfrak{a}]=-r^{2} s t \mathfrak{a}=0,} & {[r t \mathfrak{c}, s \mathfrak{b}]=-r s^{2} t \mathfrak{b}=0}
\end{array}
$$

e quindi anche l'asserto (b) è dimostrato.

Si supponga quindi $r s t \neq 0$ e si consideri il sottoanello $\mathfrak{N}$ di $\mathfrak{R}$ generato dagli elementi $r s \mathfrak{a}$, stb e $r t \mathfrak{c}$. Una semplice verifica mostra che $\mathfrak{i}$ è un ideale di $\mathfrak{R}$, che $\mathfrak{Q} / \mathfrak{M}$ è finito e che il suo ordine divide $r^{2} s^{2} t^{2}$.

Per dimostrare l'asserto è quindi sufficiente dimostrare che $\mathfrak{A}$ è finito e che il suo ordine divide $r^{3}, s^{3}$ e $t^{3}$.

Siano

- $\mathfrak{I}_{1}$ il sottoanello di $\mathfrak{N}$ generato da $r s t c ;$

- $\mathfrak{I}_{2}$ il sottoanello di $\mathfrak{N}$ generato da rsta;

- $\mathfrak{I}_{3}$ il sottoanello di $\mathfrak{N}$ generato da $r s t \mathfrak{b}$;

Utilizzando le relazioni (2) una verifica diretta porge che $\mathfrak{I}_{1}, \mathfrak{I}_{2}$ e $\mathfrak{I}_{3}$ sono ideali di $\mathfrak{N}$ (anzi di $\mathfrak{2})$.

Dalla relazione (1) si ottiene $r s \mathfrak{a}=-s t \mathfrak{b}-r t \mathfrak{c}$ e quindi $\mathfrak{N}$ è generato come anello da stb e rtc. Siccome $s(r t \mathfrak{c})=r s t \mathfrak{b} \in \mathfrak{I}_{1}$ e $s(s t \mathfrak{b})=s^{2} t \mathfrak{b}=$ $=r s t c \in \mathfrak{I}_{1}$ se ne deduce che $\mathfrak{X} / \mathfrak{I}_{1}$ è finito e che il suo ordine divide $s^{2}$. Dalla relazione (5.c) si ricava $s(r s t) \mathfrak{c}=r s^{2} t \mathfrak{c}=0$ e quindi $\mathfrak{I}_{1}$ è finito e il suo ordine divide $s$. Dunque $|\mathfrak{l}|$ (è finito e) divide $s^{3}$. 
Un ragionamento analogo applicato a $\mathfrak{I}_{2}\left(\mathrm{a} \mathfrak{I}_{3}\right)$ mostra che $\mathfrak{N}$ è anche un divisore di $t^{3}$ (di $\left.r^{3}\right)$.

Il Teorema è quindi dimostrato.

Se $r=s=t$ dalle relazioni (2) si ricava $t^{3} \mathfrak{a}=t^{3} \mathfrak{b}=t^{3} \mathfrak{c}$ e moltiplicando per $t$ la (1) (che è diventata $t^{2} \mathfrak{a}+t^{2} \mathfrak{b}+t^{2} \mathfrak{c}=0$ ) si ottiene

$$
3 t^{3} \mathfrak{a}=3 t^{3} \mathfrak{b}=3 t^{3} \mathfrak{c}=0 .
$$

Tenendo conto che (3) porge $t^{4} \mathfrak{a}=t^{4} \mathfrak{b}=t^{4} \mathfrak{c}=0$ si possono dare due casi

- 3 non divide $t$; allora $L(t, t, t)$ ha ordine $t^{8}$ ed è nilpotente di classe 3 ,

- 3 divide $t$; allora $L(t, t, t)$ ha ordine $3 t^{8}$ ed è nilpotente di classe 4 .

Si osservi che, in generale, $M(t, t, t)$ non è nemmeno nilpotente (infatti esso risulta finito e nilpotente se e solo se $t=2$ o $t=-3$ ); si può però considerare il quoziente $R M(t, t, t)$ di $M(t, t, t)$ tramite il suo residuale nilpotente (ovvero l'ultimo termine della serie centrale discendente).

TABELla 1.

\begin{tabular}{|c|c|c|c|c|}
\hline \multirow{2}{*}{$t$} & \multicolumn{2}{|c|}{$L(t, t, t)$} & \multicolumn{2}{c|}{$R M(t, t, t)$} \\
\cline { 2 - 5 } & ordine & classe & ordine & classe \\
\hline 2 & $2^{8}$ & 3 & $2^{11}$ & 4 \\
\hline $3^{n}$ & $3^{8 n+1}$ & 4 & $3^{8 n+1}$ & 4 \\
\hline$p^{n}, p^{n} \neq 2, p \neq 3$ & $p^{8 n}$ & 3 & $p^{8 n}$ & 3 \\
\hline 6 & $2^{8} \cdot 3^{9}$ & 4 & $2^{14} \cdot 3^{9}$ & 5 \\
\hline 12 & $2^{16} \cdot 3^{9}$ & 4 & $2^{16} \cdot 3^{9}$ & 4 \\
\hline 10 & $2^{8} \cdot 5^{8}$ & 3 & $2^{11} \cdot 5^{8}$ & 4 \\
\hline 20 & $2^{16} \cdot 5^{8}$ & 3 & $2^{16} \cdot 5^{8}$ & 3 \\
\hline 14 & $2^{8} \cdot 7^{8}$ & 3 & $2^{17} \cdot 7^{8}$ & 6 \\
\hline 28 & $2^{16} \cdot 7^{8}$ & 3 & $2^{16} \cdot 7^{8}$ & 3 \\
\hline 30 & $2^{8} \cdot 3^{9} \cdot 5^{8}$ & 4 & $2^{20} \cdot 3^{9} \cdot 5^{8}$ & 7 \\
\hline 60 & $2^{16} \cdot 3^{9} \cdot 5^{8}$ & 4 & $2^{16} \cdot 3^{9} \cdot 5^{8}$ & 4 \\
\hline 42 & $2^{8} \cdot 3^{9} \cdot 7^{8}$ & 4 & $2^{11} \cdot 3^{9} \cdot 7^{8}$ & 4 \\
\hline 510 & $2^{8} \cdot 3^{9} \cdot 5^{8} \cdot 17^{8}$ & 4 & $2^{32} \cdot 3^{9} \cdot 5^{8} \cdot 17^{8}$ & 11 \\
\hline
\end{tabular}


Utilizzando il software GAP e i calcoli svolti sopra si ottiene la Tabella 1. I dati riportati in tale tabella suggeriscono che (a parte il caso eccezionale in cui $t$ è il doppio di un numero dispari) vi sia una buona corrispondenza tra i due tipi di struttura.

Per l'esatto ordine di $M(t, t, t)$ e $R M(t, t, t)$ si veda [9] e l'Osservazione 3.

\section{Dimostrazione della Proposizione 3.}

L'analogo nei gruppi dell'identità di Jacobi è l'identità Hall-Witt (si veda il Teorema 2.2.3.i di [3] o il 5.1.5.iv di [12])

$$
\left[x, y^{-1}, z\right]^{y}\left[y, z^{-1}, x\right]^{z}\left[z, x^{-1}, y\right]^{x}=1,
$$

o, equivalentemente,

$$
\left[x, y, z^{x}\right]\left[z, x, y^{z}\right]\left[y, z, x^{y}\right]=1 .
$$

Questa identità, anche se non è maneggevole come quella di Jacobi, costituisce uno strumento essenziale per lo studio dei gruppi $M(r, s, t) \mathrm{e}$ dei loro quozienti. Infatti, posto $\rho=(1+r)^{|s|}-1, \sigma=(1+s)^{|t|}-1 \mathrm{e}$ $\tau=(1+t)^{|r|}-1$, si perviene al seguente risultato.

Lemma 2. Sia $G=\langle a, b, c\rangle$ un gruppo isomorfo a un quoziente di $M(r, s, t)$ con $r, s, t$ numeri interi tutti maggiori di 0. Allora si ha:

(1) $a^{(1+r) \rho} b^{(1+s) \sigma} c^{(1+t) \tau}=1$;

(2) $a^{r^{2} \rho}=1, b^{s^{2} \sigma}=1, c^{t^{2} \tau}=1$;

(3) $a^{s t \rho}=1, b^{r t \sigma}=1, c^{r s \tau}=1$;

(4) $a^{r \rho} \in\langle b\rangle, b^{s \sigma} \in\langle c\rangle, c^{t \tau} \in\langle a\rangle$.

Dim. (1) si ottiene direttamente dall'identità di Hall-Witt mentre (2) e (3) si ottengono da (1) tramite alcuni calcoli sui commutatori (per i particolari si vedano i lavori [7] e [1] nonché la dimostrazione del Lemma 7).

Per dimostrare (4) si pone $x=a^{\rho}, y=b^{\sigma}$ e $z=c^{\tau}$. Per il punto (1) si ha $x^{1+r} z^{1+t} \in\langle b\rangle$ e quindi $x^{1+r} z^{1+t}=\left(x^{1+r} z^{1+t}\right)^{b}$. Dal punto (2) discende che $x^{r^{2}}=1$, si può quindi scrivere $x^{r}=[x, b]=z^{1+t}\left(z^{-(1+t)}\right)^{b}$ e siccome $z^{1+t}\left(z^{-(1+t)}\right)^{b}=\left[z^{-(1+t)}, b\right] \in B$ se ne conclude che $x^{r} \in\langle b\rangle$. In modo analogo si prova che $y^{s} \in\langle c\rangle$ e $z^{t} \in\langle a\rangle$.

OSSERVAZIONE 3. Il Lemma 2 spiega in parte l'eccezionalità del caso $M(2,2,2)=R M(2,2,2)$. Infatti mentre in generale $t^{2}$ divide $\tau=(1+t)^{|t|}-1$ ma $t^{3}$ non lo divide, se $t=2$ si ha che $2^{3}=3^{2}-1$. 
Inoltre in [9] Mennicke ha determinato l'esatto ordine di $M(t, t, t)$ (a parte un errore nel caso un cui $t$ è pari). Si ha:

$$
|M(t, t, t)|= \begin{cases}t^{2} \tau^{3} & \text { se }(3, t)=1 \\ 3 t^{2} \tau^{3} & \text { se }(3, t)=3\end{cases}
$$

Da ciò si deduce facilmente che se $\tau=\tau_{1} \tau_{2} \operatorname{con} \pi\left(\tau_{1}\right)=\pi(t)$ e $\left(t, \tau_{2}\right)=1$ allora

$$
|R M(t, t, t)|= \begin{cases}t^{2} \tau_{1}^{3} & \text { se }(3, t)=1 \\ 3 t^{2} \tau_{1}^{3} & \text { se }(3, t)=3\end{cases}
$$

OSSERVAZIONE 4. Non è difficile dimostrare che

$$
M(r, s, t) \simeq M(s, t, r) \simeq M(t, r, s)
$$

ma, in generale, $M(r, s, t) \nsucceq M(r, t, s)$. Ad esempio $M(1,2,3)$ ha ordine 234 mentre $M(1,3,2)$ ha ordine 210 .

Oltre all'Osservazione 4 e alcune identità sui commutatori enunciate nei Lemmi 2.2.2 e 2.2.4 di [3], che saranno adoperate senza un esplicito richiamo, nella dimostrazione della Proposizione 3 si utilizzano anche i seguenti lemmi.

LEMMA 3. Sia $G$ un gruppo generato da tre suoi sottogruppi ciclici $A, B$ e C. Se $[A, B] \leq A,[B, C] \leq B e[C, A] \leq C$ allora $A \cap B, B \cap C$ e $C \cap A$ sono sottogruppi normali di $G$ e si ha $[(A \cap B)(B \cap C)(C \cap A), G] \leq A \cap B \cap C$. In particolare $A \cap B, B \cap C$ e $C \cap A$ sono contenuti in $Z_{2}(G)$ e se $A \cap B \cap C=\{1\}$ essi sono contenuti in $Z(G)$.

Dim. Il sottogruppo $A \cap B$ è centralizzato da $A$ e da $B$. Siccome $C$ normalizza $B$ e $B$ è ciclico, ne segue che $C$ normalizza $A \cap B$ e dunque $A \cap B \unlhd G$. In maniera analoga si prova che $B \cap C \unlhd G$ e $C \cap A \unlhd G$.

Se $A \cap B \cap C=\{1\}$ allora, se si procede come nella prima parte della dimostrazione del Lemma 1 , si prova che $[A \cap B, G]=\{1\},[B \cap C, G]=\{1\}$ e $[C \cap A, G]=\{1\}$. Dunque $[(A \cap B)(B \cap C)(C \cap A), G] \leq A \cap B \cap C$ e siccome $A \cap B \cap C \leq Z(G)$ l'asserto è dimostrato.

LEMMA 4. Sia $G$ un gruppo isomorfo a un quoziente di $M(r, s, t)$ con $r, s, t \in \mathbb{Z}$ tutti maggiori di 0 . Se $Z(G)=\{1\}$ allora $G$ è metabeliano.

Dim. Il Lemma 3 ed il fatto che $Z(G)=\{1\}$ porgono che

$$
A \cap B=B \cap C=C \cap A=\{1\} .
$$

Quindi, per il punto (4) del Lemma 2, $a^{r \rho}=b^{s \sigma}=c^{t \tau}=1$. Si conclude in quanto $G^{\prime}=\left\langle a^{r}, b^{s}, c^{t}\right\rangle$ e $G^{\prime \prime}=\left\langle a^{r \rho}, b^{s \sigma}, c^{t \tau}\right\rangle$. 
Lemma 5. Sia $P=\langle x\rangle$ un gruppo ciclico di ordine $p^{n}$ e sia $A$ un sottogruppo ciclico di $\operatorname{Aut}(P)$ di ordine $p^{k}$. Allora esiste un opportuno $\alpha \in A$ che genera $A$ con $x^{\alpha}=x^{\ell}$ tale che

(1) se $p>2$ allora $\ell=1+p^{n-k}$;

(2) se $p=2$ allora si possono dare $i$ seguenti casi

(i) $\ell=1+2^{n-k-1}$,

(ii) $\ell=-1+2^{n-k-1}$,

(iii) $k=1$ e $\ell=-1$.

Dim. L'asserto discende facilmente dal Lemma 5.4.1 di [3] e da semplici considerazioni aritmetiche.

È conveniente dimostrare a parte che la Proposizione 3 è valida nel caso dei $p$-gruppi.

Lemma 6. Sia $G$ un p-gruppo (finito) fattorizzato tramite tre sottogruppi ciclici $A, B$ e C tali che $[A, B] \leq A,[B, C] \leq B e[C, A] \leq C$. Allora $G^{\prime \prime} \leq Z(G)$ e $\gamma_{3}(G) \leq Z\left(G^{\prime}\right)$.

Dim. Sia $A=\langle a\rangle, B=\langle b\rangle$ e $C=\langle c\rangle$ con $a^{b}=a^{1+r}, b^{c}=b^{1+s}$ e $c^{a}=c^{1+t}$. Si osservi che, per ogni $i, j, k \in Z$, si ha $\left(a^{i}\right)^{b}=\left(a^{i}\right)^{1+r},\left(b^{j}\right)^{c}=\left(b^{j}\right)^{1+s} \mathrm{e}$ $\left(c^{k}\right)^{a}=\left(c^{k}\right)^{1+t}$. Si può quindi supporre (rimpiazzando eventualmente $a, b$ e $c$ con opportune loro potenze) che gli automorfismi indotti per coniugio da $a$, $b$ e $c$ rispettivamente su $C, A$ e $B$ abbiano la forma descritta dal Lemma 5.

Se $K \in \mathbb{N}$ e $K= \pm p^{n}+H p^{n+1}$ (con $H, n \in \mathbb{N}$ ) si scriverà $K= \pm p^{n}+$ $+\vartheta(n)$ e, quando non vi sia possibilità di confusione, $K= \pm p^{n}+\vartheta$.

Tenendo presente che

$$
G^{\prime}=\left\langle a^{r}, b^{s}, c^{t}\right\rangle, \quad G^{\prime \prime}=\left\langle a^{r \rho}, b^{s \sigma}, c^{t \tau}\right\rangle
$$

$\mathrm{e}$

$$
[G, G, G]=\left\langle a^{r^{2}}, b^{s^{2}}, c^{t^{2}}, a^{\rho}, b^{\sigma}, c^{\tau}\right\rangle
$$

si devono considerare vari casi.

$$
\mathbf{I}^{\mathbf{o}} \text { Caso. } r=p^{\alpha}, s=p^{\beta} \text { e } t=p^{\gamma}(\alpha, \beta, \gamma \in \mathbb{N}) .
$$

Si può supporre $\alpha \leq \beta \leq \gamma$ (il caso $\alpha \leq \gamma \leq \beta$ si tratta in maniera ana$\operatorname{loga)}$.

Con le notazioni introdotte sopra si ha

$$
\rho=p^{\alpha+\beta}+\vartheta, \quad \sigma=p^{\beta+\gamma}+\vartheta \text { e } \tau=p^{\alpha+\gamma}+\vartheta .
$$


Dalle relazioni (2) e (3) del Lemma 2 si ricava che l'ordine di $a$ divide $p^{3 \alpha+\beta}$, quello di $b$ divide $p^{3 \beta+\gamma}$ e $p^{\alpha+\beta+2 \gamma}$ e quello di $c$ divide $p^{2 \alpha+\beta+\gamma}$.

Dal Lemma 2 discende $\left[a^{r \rho}, b\right]=\left[b^{s \sigma}, c\right]=\left[c^{t \tau}, a\right]=1$.

Poi $\left[c, a^{r \rho}\right]=c^{(1+t)^{r \rho}-1}$ e siccome $(1+t)^{r \rho}-1=p^{2 \alpha+\beta+\gamma}+\vartheta$ si ha $\left[c, a^{r \rho}\right]=1$. Analogamente $(1+r)^{s \sigma}-1=p^{\alpha+2 \beta+\gamma}+\vartheta$ e siccome per ipotesi $3 \alpha+\beta \leq \alpha+2 \beta+\gamma$ si ha $\left[a, b^{s \sigma}\right]=1$. Infine $(1+s)^{t \tau}-1=p^{\alpha+\beta+2 \gamma}+\vartheta \mathrm{e}$ $\left[b, c^{t \tau}\right]=1$ porge che $G^{\prime \prime} \leq Z(G)$.

Si ha $\left[a^{r^{2}}, b^{s}\right]=a^{r^{2} \rho}=1,\left[b^{s^{2}}, c^{t}\right]=b^{s^{2} \sigma}=1$ e $\left[c^{t^{2}}, a^{r}\right]=c^{t^{2} \tau}=1$. Poi

- $\left[c^{t}, a^{r^{2}}\right]=c^{t\left\{(1+t)^{r^{2}}-1\right\}}$, si ha $t\left\{(1+t)^{\gamma^{2}}-1\right\}=p^{2 \alpha+2 \gamma}+\vartheta$ e siccome $2 \alpha+\beta+\gamma \leq 2 \alpha+2 \gamma$ risulta $\left[c^{t}, a^{r^{2}}\right]=1$

- $\left[a^{r}, b^{s^{2}}\right]=a^{r\left\{(1+r)^{s^{2}}-1\right\}}$, si ha $r\left\{(1+r)^{s^{2}}-1\right\}=p^{2 \alpha+2 \beta}+\vartheta$ e siccome $3 \alpha+\beta \leq 2 \alpha+2 \beta$ risulta $\left[a^{r}, b^{s^{2}}\right]=1$;

- $\left[b^{s}, c^{t^{2}}\right]=b^{s\left\{(1+s)^{t^{2}}-1\right\}}$, si ha $s\left\{(1+s)^{t^{2}}-1\right\}=p^{2 \beta+2 \gamma}+\vartheta$ e siccome $\alpha+\beta+2 \gamma \leq 2 \beta+2 \gamma$ risulta $\left[b^{s}, c^{t^{2}}\right]=1$;

- $\left[\alpha^{\rho}, b^{s}\right]=a^{\rho^{2}}, \rho^{2}=p^{2 \alpha+2 \beta}+\vartheta, 3 \alpha+\beta \leq 2 \alpha+2 \beta$ e quindi $\left[\alpha^{\rho}, b^{s}\right]=1$;

- $\left[c^{t}, a^{\rho}\right]=c^{t\left\{(1+t)^{\rho}-1\right\}}, t\left\{(1+t)^{\rho}-1\right\}=p^{\alpha+\beta+2 \gamma}+\vartheta, 2 \alpha+\beta+\gamma \leq \alpha+\beta+2 \gamma$ e quindi $\left[c^{t}, \alpha^{\rho}\right]=1$;

- $\left[b^{\sigma}, c^{t}\right]=b^{\sigma^{2}}, \sigma^{2}=p^{2 \alpha+2 \beta}+\vartheta, \alpha+\beta+2 \gamma \leq 2 \alpha+2 \beta$ e quindi $\left[b^{\sigma}, c^{t}\right]=1$;

- $\left[a^{r}, b^{\sigma}\right]=a^{r\left\{(1+r)^{\sigma}-1\right\}}, r\left\{(1+r)^{\sigma}-1\right\}=p^{2 \alpha+\beta+\gamma}+\vartheta, 3 \alpha+\beta \leq 2 \alpha+\beta+\gamma$ e quindi $\left[a^{r}, b^{\sigma}\right]=1$;

- $\left[c^{\tau}, a^{r}\right]=c^{\tau^{2}}, \tau^{2}=p^{2 \alpha+2 \gamma}+\vartheta, 2 \alpha+\beta+\gamma \leq 2 \alpha+2 \gamma$ e quindi $\left[c^{\tau}, a^{t}\right]=1$;

- la dimostrazione che $\left[b^{s}, c^{\tau}\right]=1$ richiede un trattamento diverso. Dalla relazione (1) del Lemma 2 si ricava $c^{-\tau}=a^{(1+r) \rho} b^{(1+s) \sigma} c^{t \tau}$. Si ha $\left[b^{s}, a^{\rho}\right]=a^{-\rho^{2}}=1$ (per quanto visto sopra) e $\left[b^{s}, c^{t \tau}\right]=1$ perché $s\left\{(1+s)^{t \tau}-1\right\}=p^{\alpha+2 \beta+2 \gamma}+\vartheta$ e $\alpha+\beta+2 \gamma \leq \alpha+2 \beta+2 \gamma$. Ovviamente $b^{s}$ commuta con $b^{(1+s) \sigma}$ e quindi $\left[b^{s}, c^{\tau}\right]=1$.

II $^{\mathbf{0}}$ Caso. $p=2$ e $1+r= \pm 1+2^{\alpha}, 1+s= \pm 1+2^{\beta}$ e $1+t= \pm 1+2^{\gamma}$ $(\alpha, \beta, \gamma \in \mathbb{N})$.

Questo caso comprende anche il caso (iii) del Lemma 5 (se, ad esempio, $b$ induce l'inversione su $A$ si può sempre scrivere $\left.a^{b}=a^{-1+2^{|A|}}\right)$.

Anche in questo caso si può supporre $\alpha \leq \beta \leq \gamma$ in quanto l'altra possibilità si tratta in maniera analoga. A titolo esemplificativo viene considerato solamente il caso

$$
1+r=-1+2^{\alpha}, \quad 1+s=1+2^{\beta} \text { e } 1+t=1+2^{\gamma}
$$

in quanto gli altri casi sono del tutto simili. 
Si ha

$$
r=-2+2^{\alpha}=-2+\vartheta, \quad s=2^{\beta} \text { e } t=2^{\gamma}
$$

da cui

$$
\rho=2^{\beta+1}+\vartheta, \quad \sigma=2^{\beta+\gamma}+\vartheta \text { e } \tau=2^{\gamma+1}+\vartheta .
$$

Dal Lemma 2 si ottiene che l'ordine di $a$ divide $2^{\beta+3}$, quello di $b$ divide $2^{3 \beta+\gamma}$ e $2^{\beta+2 \gamma+1}$ e quello di $c$ divide $2^{\beta+\gamma+2}$. Da $(1+t)^{r \rho}-1=2^{\beta+\gamma+2}+\vartheta$ si ha $\left[c, a^{r \rho}\right]=1$. Da $(1+r)^{s \sigma}-1=-2^{\alpha+2 \beta+\gamma}+\vartheta$ e $\beta+3 \leq \alpha+2 \beta+\gamma$ si ha $\left[a, b^{s \sigma}\right]=1$. Infine da $(1+s)^{t \tau}-1=2^{\beta+\gamma+2}+\vartheta$ e $\beta+\gamma+2 \leq \beta+2 \gamma+1$ si ottiene $\left[b, c^{t \tau}\right]=1$.

Questo prova che $G^{\prime \prime} \leq Z(G)$; il fatto che $[G, G, G] \leq Z\left(G^{\prime}\right)$ si dimostra in maniera analoga.

Dimostrazione della Proposizione 3. Sia $A=\langle a\rangle, B=\langle b\rangle$ e $C=\langle c\rangle$.

Conviene iniziare dimostrando che $G$ è supersolubile. Ragionando come nella dimostrazione della Proposizione 1 si ricava che se $A_{G}, B_{G}$ e $C_{G}$ sono tutti identici allora $G$ è nilpotente e quindi (essendo finitamente generato) supersolubile. Esclusa tale possibilità, si devono distinguere quattro casi.

(a) I tre sottogruppi $A, B$ e $C$ sono finiti.

In questo caso si ragiona per induzione su $|A|+|B|+|C|$ (la base dell'induzione è triviale). Se $A_{G} \neq\{1\}$, l'ipotesi induttiva applicata a $G / A_{G}$ ed il fatto che $A_{G}$ è ciclico porgono la conclusione. Si ragiona in maniera analoga se $B_{G} \neq\{1\}$ o $C_{G} \neq\{1\}$.

(b) Uno solo tra i gruppi $A, B$ e $C$ è infinito.

Non è restrittivo supporre che sia $C$ ad avere cardinalità infinita; in tal caso si ragiona per induzione su $|A|+|B|$. Se $A_{G} \neq\{1\}$ (o $B_{G} \neq\{1\}$ ) l'ipotesi induttiva applicata a $G / A_{G}$ (ovvero a $G / B_{G}$ ) e il fatto che $A_{G}$ (e $\left.B_{G}\right)$ è ciclico permettono di concludere. Se $C_{G} \neq\{1\}$ allora in $\bar{G}=G / C_{G}$ anche $\bar{C}$ risulta finito e si conclude per il punto precedente.

(c) Uno solo tra i sottogruppi $A, B$ e $C$ è finito.

Si supponga che sia $A$ ad essere finito. In questo caso si ragiona per induzione su $|A|$. Se $A_{G} \neq\{1\}$ si conclude per l'ipotesi induttiva, se $B_{G} \neq\{1\}$ (oppure $C_{G} \neq\{1\}$ ) allora, considerando $G / B_{G}$ (oppure $G / C_{G}$ ), si è ricondotti al caso (b).

(d) I tre sottogruppi $A, B$ e $C$ sono infiniti. 
Allora quozientando $G$ tramite $A_{G}$ (ovvero $B_{G}$ o $C_{G}$ ) si è ricondotti al caso precedente.

Per dimostrare che $G^{\prime \prime} \leq Z(G)$ e $\gamma_{3}(G) \leq Z\left(G^{\prime}\right)$ si devono distinguere cinque casi (tenendo conto del fatto che gli unici automorfismi del gruppo ciclico infinito sono l'identità e l'inversione).

(1) $[a, b]=1($ o $[b, c]=1$ o $[c, a]=1)$.

In questo caso il gruppo $A B$ è abeliano e $G$ si fattorizza nel prodotto dei due sottogruppi abeliani $A B$ e $C$. Per un noto risultato dovuto a Itô ([5]) $G$ risulta metabeliano e in questo caso l'asserto è dimostrato.

(2) $a^{b}=a^{-1}, b^{c}=b^{-1}$ e $c^{a}=c^{-1}$.

In questo caso si ha $G^{\prime}=\left\langle a^{2}, b^{2}, c^{2}\right\rangle$ e si verifica facilmente che $G^{\prime}$ risulta abeliano.

(3) $a^{b}=a^{-1}, b^{c}=b^{-1}, c^{a}=c^{1+t}, b$ ha ordine infinito e $c$ ha ordine finito.

Dall'identità di Hall-Witt si ottiene $\left[a, b, c^{a}\right]\left[c, a, b^{c}\right]\left[b, c, a^{b}\right]=1$ da cui $\left[a^{-2}, c\right]^{a}\left[c^{t}, b\right]^{c}\left[b^{-2}, a\right]^{b}=1$ e $\left(c^{(1+t)^{2}-1}\right)^{a^{-1}}=\left(b^{-1+(-1)^{t}}\right)^{c}$. Siccome per ipotesi $c$ ha ordine finito e $b$ ha ordine infinito i due membri dell'ultima uguaglianza devono ridursi all'identità e quindi $\left[c, a^{2}\right]=1 \mathrm{e}\left[b, c^{t}\right]=1$. Si ha ovviamente $\left[a, b^{2}\right]=1$ e quindi $G^{\prime}=\left\langle a^{2}, b^{2}, c^{t}\right\rangle$ risulta abeliano.

(4) $a^{b}=a^{-1}, b^{c}=b^{1+s}, c^{a}=c^{1+t}, b$ e $c$ hanno ordine finito e $a$ ha ordine infinito.

Siccome $b$ e $c$ hanno ordine finito si può supporre $s \geq 1$ e $t \geq 1$. Da $\left[a, b, c^{a}\right]\left[c, a, b^{c}\right]\left[b, c, a^{b}\right]=1$ segue $\left[a^{-2}, c\right]^{a}\left[c^{t}, b\right]^{c}\left[b^{s}, a\right]^{b}=1$ e $\left[a, b^{s}\right] \in B C$. $\mathrm{Ma}\left[a, b^{s}\right]=a^{-1+(-1)^{s}}$ e $a$ ha ordine infinito mentre $B C$ è un sottogruppo finito di $G$, dunque $\left[a, b^{s}\right]=1$. Si ha poi $\left[c, a^{2}\right]^{a^{-1}}=\left(b^{\sigma}\right)^{c}$ (ove $\sigma=(1+s)^{|t|}-1$ ). Poiché $A \cap B \cap C=\{1\}$ il Lemma 3 porge $B \cap C \leq Z(G)$ e quindi $b^{\sigma} \in Z(G) \mathrm{e}$ $\left[c, a^{2}\right]=c^{2 t+t^{2}} \in Z(G)$. Si ha $b^{\sigma}=\left(b^{\sigma}\right)^{c}=b^{(1+s) \sigma}$ da cui $b^{s \sigma}=1$ e quindi $\left[b^{s}, c^{t}\right]=1$. Analogamente $c^{2 t+t^{2}}=\left(c^{2 t+t^{2}}\right)^{a}=c^{(1+t)\left(2 t+t^{2}\right)}$ porge $\left[c^{t}, a^{2}\right]=1$. Siccome $\left[a^{2}, b^{s}\right]=1$ ne risulta che $G^{\prime}=\left\langle a^{2}, b^{s}, c^{t}\right\rangle$ è abeliano.

(5) $a^{b}=a^{1+r}, b^{c}=b^{1+s}, c^{a}=c^{1+t}$, con $a, b$ e $c$ di ordine finito.

Non è restrittivo supporre $r, s, t \geq 1$. Inoltre in questo caso $G$ ha ordine finito; per provare l'asserto si ragiona per induzione su $|G|$ (la base dell'induzione essendo triviale).

Siano $N_{1}$ e $N_{2}$ due sottogruppi normali minimali distinti di $G$. Allora, posto $G_{1}=G / N_{1}$ e $G_{2}=G / N_{2}$, dall'ipotesi induttiva si ricava

$$
\left[G_{i}^{\prime \prime}, G_{i}\right]=\{1\}=\left[\left[G_{i}, G_{i}, G_{i}\right], G_{i}^{\prime}\right] \quad(i \in\{1,2\})
$$


da cui $\left[G^{\prime \prime}, G\right] \leq N_{1} \cap N_{2}=\{1\}$, $\left[[G, G, G], G^{\prime}\right] \leq N_{1} \cap N_{2}=\{1\}$ e in questo caso l'asserto è provato.

Quindi si può supporre che $G$ ammetta un unico sottogruppo normale minimo $N$; tale $N$ risulta essere un $p$-gruppo per qualche numero primo $p$. Ne discende che $F=F(G)$, il sottogruppo di Fitting di $G$, è un $p$-sottogruppo di $G$. Inoltre, essendo $G$ supersolubile, si ha $G^{\prime} \leq F$ (5.4.10 di [12]) e $F$ è un $p$-sottogruppo di Sylow di $G$.

Si può scrivere $a=a_{1} a_{2}, b=b_{1} b_{2}$ e $c=c_{1} c_{2}$ con $a_{1}, b_{1}$ e $c_{1} p$-elementi di $G$ (necessariamente contenuti in $F$ ) e $a_{2}, b_{2}$ e $c_{2}$ elementi di ordine coprimo con $p$. Si ha $F=\left\langle a_{1}, b_{1}, c_{1}\right\rangle$ e posto $H=\left\langle a_{2}, b_{2}, c_{2}\right\rangle$ si verifica (tenendo conto che $b_{2}$ normalizza $\left\langle a_{2}\right\rangle, c_{2}$ normalizza $\left\langle b_{2}\right\rangle$ e $a_{2}$ normalizza $\left.\left\langle c_{2}\right\rangle\right)$ che $H$ è un $p^{\prime}$-sottogruppo di $G$; si ha quindi $G=F H, F \cap H=\{1\}$. Inoltre, poiché $G / F$ è abeliano, anche $H$ risulta abeliano.

Sia $c_{2} \neq 1$. Si ha $\left[H, c_{2}\right]=\{1\}$ e $\left[c_{1}, c_{2}\right]=1$, inoltre $\left[c_{2}, a_{1}\right] \in$ $\in F \cap\left\langle c_{2}\right\rangle=\{1\}$. Quindi $\left[G, c_{2}\right]=\left\langle\left[b_{1}, c_{2}\right]\right\rangle$ e siccome $\left\langle b_{1}\right\rangle$ è un $p$-gruppo mentre $c_{2}$ induce un $p^{\prime}$-automorfismo su $\left\langle b_{1}\right\rangle$ si ha $\left[\left\langle\left[b_{1}, c_{2}\right]\right\rangle, c_{2}\right]=\left\langle\left[b_{1}, c_{2}\right]\right\rangle$.

Non si può avere $\left[b_{1}, c_{2}\right]=1$ perché in tal caso $c_{2} \in Z(G) \leq F$. Quindi $\left\langle\left[b_{1}, c_{2}\right]\right\rangle$ deve contenere $N$, l'unico sottogruppo minimale di $G$, e siccome $\left\langle\left[b_{1}, c_{2}\right]\right\rangle \cap Z(G)=\{1\}$ si deve avere $Z(G)=\{1\}$. Dal Lemma 4 discende che $G$ è metabeliano e in questo caso l'asserto è dimostrato.

Si conclude allo stesso modo se $a_{2} \neq 1$ o $b_{2} \neq 1$. Infine, nel caso in cui $a_{2}=b_{2}=c_{2}=1, G=F$ è un $p$-gruppo (finito) e il Lemma 6 porge la conclusione.

\section{Dimostrazione del Teorema 1.}

È conveniente enunciare la seguente generalizzazione del Lemma 2.

Lemma 7. Siano $r, s, t \in Z$ tutti diversi da 0 e da -2 e sia $G=\langle a, b, c\rangle$ un quoziente di $M(r, s, t)=\left\langle a_{*}, b_{*}, c_{*} \mid a_{*}^{b_{*}}=a_{*}^{1+r}, b_{*}^{c_{*}}=b_{*}^{1+s}, c_{*}^{a_{*}}=c_{*}^{1+t}\right\rangle$, allora $G$ è finito e risulta

(1) $\left\langle a^{\rho}\right\rangle \leq\left\langle b^{\sigma}, c^{\tau}\right\rangle,\left\langle b^{\sigma}\right\rangle \leq\left\langle a^{\rho}, c^{\tau}\right\rangle e\left\langle c^{\tau}\right\rangle \leq\left\langle a^{\rho}, b^{\sigma}\right\rangle$;

(2) $a^{r \rho} \in\langle b\rangle, b^{s \sigma} \in\langle c\rangle, c^{t \tau} \in\langle a\rangle$;

(3) $a^{r^{2} \rho}=1, b^{s^{2} \sigma}=1, c^{t^{2} \tau}=1$;

(4) $a^{s \rho} \in\langle c\rangle, b^{t \sigma} \in\langle a\rangle, c^{r \tau} \in\langle b\rangle$;

(5) $a^{s t \rho}=1, b^{r t \sigma}=1, c^{r s \tau}=1$.

Dim. Utilizzando l'identità di Hall-Witt $\left[a, b, c^{a}\right]\left[c, a, b^{c}\right]\left[b, c, a^{b}\right]=1$ si 
perviene a

$$
\left[a^{r}, c^{a}\right]\left[c^{t}, b^{c}\right]\left[b^{s}, a^{b}\right]=1
$$

da cui

$$
\left[a, b^{s}\right]^{b}\left[b, c^{t}\right]^{c}\left[c, a^{r}\right]^{a}=1 .
$$

Se $s>0$ allora $\left[a, b^{s}\right]=a^{\rho}$; se invece $s<0$ allora $\left[a, b^{s}\right]=\left[a, b^{-s}\right]^{-b^{s}}=$ $=\left(a^{\rho}\right)^{-b^{s}}$. Analogamente se $t>0$ allora $\left[b, c^{t}\right]=b^{\sigma}$, se $t<0$ allora $\left[b, c^{t}\right]=\left[b, c^{-t}\right]^{-c^{t}}=\left(b^{\sigma}\right)^{-c^{t}}$ e se $r>0$ allora $\left[c, a^{r}\right]=c^{\tau}$ mentre se $r<0$ allora $\left[c, a^{r}\right]=\left[c, a^{-r}\right]^{-a^{r}}=\left(c^{\tau}\right)^{-a^{r}}$. Sia $a^{-b^{1+s}}=a^{i}, b^{-c^{1+t}}=b^{j}$ e $c^{-a^{1+r}}=c^{k}$ allora, posto

$$
\lambda=\left\{\begin{array}{ll}
1+r & \text { se } s>0 \\
i & \text { se } s<0
\end{array} \quad \mu=\left\{\begin{array}{ll}
1+s & \text { se } t>0 \\
j & \text { se } t<0
\end{array} \quad v= \begin{cases}1+t & \text { se } r>0 \\
k & \text { se } t<0\end{cases}\right.\right.
$$

si ottiene

$$
a^{\lambda \rho} b^{\mu \sigma} c^{v \tau}=1
$$

Per dimostrare che $G$ è finito si devono considerare due casi.

- Almeno uno tra $a, b$ e $c$ ha ordine finito.

Non è restrittivo supporre che sia $a$ ad avere ordine finito; sia $|\langle a\rangle|=m$. Allora $c=c^{a^{m}}=c^{(1+t)^{m}}$ e quindi (siccome $1+t \neq \pm 1$ ), $c$ ha ordine finito che divide $(1+t)^{m}-1$. Se $|\langle c\rangle|=n$, ragionando in maniera analoga, si prova che $b$ ha ordine finito che divide $(1+s)^{n}-1$. Siccome ogni elemento di $G$ si può scrivere nella forma $a^{i} b^{j} c^{k}$ (con $i, j, k \in \mathbb{Z}$ opportuni) si può concludere che, in questo caso, $G$ ha ordine finito.

- I tre elementi $a, b$ e $c$ hanno tutti ordine infinito.

Ragionando per assurdo si dimostra che questo caso non si può dare. Posto $a_{1}=a^{\lambda \rho}, b_{1}=b^{\mu \sigma}$ e $c_{1}=c^{\nu \tau}$ si ha $a_{1} \neq 1, b_{1} \neq 1, c_{1} \neq 1$ e, per (西), $a_{1} b_{1} c_{1}=1$. Si osservi che non è restrittivo supporre $\lambda \rho>0$ (in caso contrario, in luogo di $a_{1}, b_{1}$ e $c_{1}$, si considerano i rispettivi inversi). Si ottiene quindi

$$
b_{1}^{-1} a_{1}^{-1}=c_{1}=c_{1}^{c}=\left(b_{1}^{-1} a_{1}^{-1}\right)^{c}=b_{1}^{-1-s} a_{1}^{-c}
$$

e $\left[a_{1}, c\right]=b_{1}^{-s}$. Siccome $a_{1}=a^{\lambda \rho}$ risulta $\left[a_{1}, c\right]=c^{1-(1+t)^{\lambda \rho}} \in\langle c\rangle$ e dunque l'elemento $b_{2}=b_{1}^{s}$ appartiene a $\langle b\rangle \cap\langle c\rangle$. Ma allora

$$
b_{2}=b_{2}^{c}=b_{2}^{(1+s)}
$$

e $b$ dovrebbe avere ordine finito, contraddicendo le ipotesi. 
Tenendo presente che $a, b$ e $c$ hanno ordine finito si ottiene

$$
\left\langle a^{\lambda \rho}\right\rangle=\left\langle a^{\rho}\right\rangle, \quad\left\langle b^{\mu \sigma}\right\rangle=\left\langle b^{\sigma}\right\rangle \text { e }\left\langle c^{\nu \tau}\right\rangle=\left\langle c^{\tau}\right\rangle ;
$$

utilizzando tali uguaglianze si dimostra facilmente (1).

Il punto (2) si ricava ragionando come nella dimostrazione del punto (4) del Lemma 2.

Dal punto (2) si ottiene

$$
1=\left[a^{r \rho}, b\right]=a^{r^{2} \rho}, 1=\left[b^{s \sigma}, c\right]=b^{s^{2} \sigma} \text { e } 1=\left[c^{t \tau}, a\right]=c^{t^{2} \tau},
$$

il che prova il punto (3).

Tenendo conto che $G$ è finito, la Proposizione 3 porge che $a^{\rho}, b^{\sigma}$ e $c^{\tau}$ commutano tra loro. Per (1) si può scrivere $a^{\rho}=b^{i \sigma} c^{j \tau}(i, j \in Z$ opportuni) e quindi $a^{s \rho}=\left(b^{i \sigma} c^{j \tau}\right)^{s}=b^{i s \sigma} c^{j s \tau}$. Poiché, per (2), $b^{s \sigma} \in\langle c\rangle$ si può concludere che $a^{s \rho} \in\langle c\rangle$. In maniera del tutto analoga si prova che $b^{t \sigma} \in\langle a\rangle$ e $c^{r \tau} \in\langle b\rangle$.

Infine, per dimostrare (5), si può scrivere $a^{s \rho}=c^{k}\left(k \in Z_{1}\right.$ opportuno) da cui $a^{s \rho}=\left(c^{k}\right)^{a}=c^{k(1+t)}=a^{(1+s) t \rho}$ e $a^{s t \rho}=1$. Allo stesso modo si verifica che $b^{r t \sigma}=1$ e $c^{r s \tau}=1$.

Dimostrazione Del Teorema 1 . Sia $G$ un quoziente di $M(r, s, t)$ con $r, s, t \in Z$. Il fatto che, se $r \neq 0,-2, s \neq 0,-2$ e $t \neq 0,-2, G$ è finito discende dal Lemma 7 (la dimostrazione fornita in [7], dove viene esplicitato solo il caso $r, s, t \in \mathbb{N}$, non è molto chiara). I fatti che $G$ è supersolubile, che $G^{\prime \prime} \leq Z(G) \mathrm{e}$ che $\gamma_{3}(G) \leq Z\left(G^{\prime}\right)$ discendono direttamente dalla Proposizione 3. Per dimostrare che, sotto tali ipotesi, l'ordine di $G$ divide $|(r, s)(s, t)(t, r) \rho \sigma \tau|$ si ragiona per induzione su $|G|$ (la base dell'induzione essendo triviale).

Il gruppo $L=\left\langle a^{\rho}, b^{\sigma}, c^{\tau}\right\rangle$ è un sottogruppo di $G$ contenuto in $\gamma_{3}(G)$, in particolare $L$ è abeliano. Inoltre $L \unlhd G$ infatti $\left[a^{\rho}, b\right]=a^{r \rho} \in L$ poi, siccome $r$ divide $\rho$, si può scrivere $\rho=r k$ e poiché $\left[c, a^{r}\right]=c^{\tau}$ e $c^{\tau}$ commuta con $c$ e con $a^{r}$ (in quanto $a^{r} \in G^{\prime}$ ) si ha $\left[a^{\rho}, c\right]=\left[c, a^{r k}\right]^{-1}=\left[c, a^{r}\right]^{-k}=c^{-k \tau} \in L$. In maniera analoga si prova che $\left[b^{\sigma}, a\right],\left[b^{\sigma}, c\right],\left[c^{\tau}, a\right],\left[c^{\tau}, b\right] \in L$.

Se $L=\{1\}$ allora l'ordine di $G$ divide $|\rho \sigma \tau|$ e l'asserto è dimostrato. Si può quindi assumere che $L \neq\{1\}$. Sia $p$ un divisore primo dell'ordine di $L$. Se $L$ non è un $p$-gruppo si può scrivere $L=L_{1} \times L_{2}$ con $L_{1} p$-sottogruppo non banale e $L_{2} p^{\prime}$-sottogruppo non banale. Per l'ipotesi induttiva gli ordini dei due gruppi $G / L_{1}$ e $G / L_{2}$ dividono entrambi $|(r, s)(s, t)(t, r) \rho \sigma \tau|$ e siccome $\left(\left|L_{1}\right|,\left|L_{2}\right|\right)=1$ anche l'ordine di $G$ deve dividere tale numero.

Si supponga quindi che $L$ sia un $p$-gruppo. Poiché $G / L$ ha ordine che divide $|\rho \sigma \tau|$ per dimostrare l'asserto è sufficiente far vedere che l'ordine di $L$ divide $(r, s)(s, t)(t, r)$; per far questo si sfrutta il fatto che il reticolo dei sottogruppi di un $p$-gruppo ciclico è totalmente ordinato. 
Sia $r=p^{\alpha} r^{\prime}, s=p^{\beta} s^{\prime}, t=p^{\gamma} t^{\prime}$ con $\left(r^{\prime} s^{\prime} t^{\prime}, p\right)=1$; si può supporre (eventualmente rinominando gli elementi $a, b$ e $c$ ) che $\alpha \leq \beta \leq \gamma$ oppure che $\alpha \leq \gamma \leq \beta$. Si può inoltre considerare solamente il caso $\alpha \leq \beta \leq \gamma$ in quanto l'altro caso è del tutto simile. Siccome $(r, s)=p^{\alpha}\left(r^{\prime}, s^{\prime}\right),(s, t)=p^{\beta}\left(s^{\prime}, t^{\prime}\right)$ e $(t, r)=p^{\alpha}\left(t^{\prime}, r^{\prime}\right)$ sarà sufficiente dimostrare che $|L|$ divide $p^{2 \alpha+\beta}$.

Dal Lemma 7 si ottiene $L=\left\langle a^{\rho}, b^{\sigma}\right\rangle$.

Ancora dal Lemma 7 si ottiene $a^{p^{2 \alpha} \rho}=1$ da cui $a^{p^{\alpha+\beta} \rho}=1$ e $a^{r s \rho}=1$; poiché $c^{r s \tau}=1$ risulta anche $b^{r s \sigma}=1$.

Sempre per il Lemma 7 si ha $a^{r \rho} \in A \cap B$; a questo punto si possono dare due casi

- $\left\langle a^{r \rho}\right\rangle \leq\left\langle b^{s \sigma}\right\rangle$.

Siccome $a^{r \rho}, b^{s \sigma} \in\left\langle b^{s \sigma}\right\rangle$ l'ordine di $L /\left\langle b^{s \sigma}\right\rangle$ divide $p^{\alpha+\beta}$ e poiché $b^{r s \sigma}=1$ l'ordine di $L$ divide $p^{2 \alpha+\beta}$.

- $\left\langle a^{r \rho}\right\rangle \geq\left\langle b^{s \sigma}\right\rangle$.

Siccome $a^{r \rho}, b^{s \sigma} \in\left\langle a^{r \rho}\right\rangle$ l'ordine di $L /\left\langle a^{r \rho}\right\rangle$ divide $p^{\alpha+\beta}$ e poiché $a^{r^{2} \sigma}=1$ l'ordine di $L$ divide $p^{2 \alpha+\beta}$.

Dunque in ogni caso $|L|$ divide $(r, s)(s, t)(t, r)$ e l'asserto è dimostrato.

Osservazione 5. Sia $G=M(r, s, t)$ e si supponga che $G$ sia finito. Se $p$ è il più piccolo divisore primo di $|G|$, siccome $G$ è supersolubile esso ammette un $p$-complemento normale (si veda 5.4.8 di [12]). In particolare essendo

$$
G / G^{\prime} \simeq C_{r} \times C_{s} \times C_{t}
$$

se $r=p^{\alpha}, s=p^{\beta}$ e $t=p^{\gamma}$ allora $R M(r, s, t)$ (il quoziente di $G$ tramite il suo residuale nilpotente) è un $p$-gruppo isomorfo a un $p$-sottogruppo di Sylow di $G$. Inoltre il residuale nilpotente $N$ di $G$ è abeliano e non è difficile dimostrare che il suo ordine è $|\rho \sigma \tau|_{p^{\prime}}$ (se $n \in \mathbb{N}$ con $n_{p^{\prime}}$ si indica la $p^{\prime}$-parte di $n$ cioè quel numero $n^{\prime}$ tale che $n=p^{\lambda} n^{\prime}$ con $\left(n^{\prime}, p\right)=1$ mentre con $n_{p}=p^{\lambda}$ si indica la $p$-parte di $n$ ).

L'esatta determinazione dell'ordine $\omega=\omega_{p}(\alpha, \beta, \gamma)$ di $R M\left(p^{\alpha}, p^{\beta}, p^{\gamma}\right)$ sembra però abbastanza difficile. Numerosi esperimenti condotti col software GAP portano a congetturare che $\omega$ divida sempre il numero

$$
\Omega=\Omega_{p}(\alpha, \beta, \gamma)= \begin{cases}\left(p^{\alpha}, p^{\beta}\right)\left(p^{\beta}, p^{\gamma}\right)\left(p^{\gamma}, p^{\alpha}\right)[\rho \sigma \tau]_{p} /\left(p^{\alpha}, p^{\beta}, p^{\gamma}\right) & \text { se } p \neq 3 \\ 3\left(3^{\alpha}, 3^{\beta}\right)\left(3^{\beta}, 3^{\gamma}\right)\left(3^{\gamma}, 3^{\alpha}\right)[\rho \sigma \tau]_{3} /\left(3^{\alpha}, 3^{\beta}, 3^{\gamma}\right) & \text { se } p=3\end{cases}
$$

Per l'Osservazione 3 si ha $\omega_{p}(\gamma, \gamma, \gamma)=\Omega_{p}(\gamma, \gamma, \gamma)$ ma, in generale, può accadere che $\omega \neq \Omega$ come mostrano i risultati riportati nella Tabella 2. Si osservi che in tutti i casi considerati si ha $\omega_{p}(\alpha, \beta, \gamma)=\omega_{p}(\alpha, \gamma, \beta)$; risulta però (ad esempio) $|M(2,4,8)|=2^{16} \cdot 3 \cdot 13 \cdot 313$ mentre $|M(2,8,4)|=2^{16} \cdot 3 \cdot 5^{2} \cdot 41^{2}$. 
Un altro problema che sembra di non facile soluzione è la determinazione dell'esatta classe di nilpotenza di $R M(r, s, t)$.

TABELla 2.

\begin{tabular}{|c|c|c|c|c|c|c|c|c|c|c|c|}
\hline & & & \multicolumn{3}{|c|}{$p=2$} & \multicolumn{3}{|c|}{$p=3$} & \multicolumn{3}{|c|}{$p=5$} \\
\cline { 5 - 13 }$\alpha$ & $\beta$ & $\gamma$ & $\omega$ & $\Omega / \omega$ & classe & $\omega$ & $\Omega / \omega$ & classe & $\omega$ & $\Omega / \omega$ & classe \\
\hline 1 & 1 & 1 & $2^{11}$ & 1 & 4 & $3^{9}$ & 1 & 5 & $5^{8}$ & 1 & 3 \\
\hline 1 & 1 & 2 & $2^{12}$ & 1 & 5 & $3^{11}$ & 1 & 5 & $5^{10}$ & 1 & 4 \\
\hline 1 & 2 & 2 & $2^{14}$ & 1 & 6 & $3^{13}$ & 3 & 5 & $5^{13}$ & 1 & 5 \\
\hline 2 & 2 & 2 & $2^{16}$ & 1 & 3 & $3^{17}$ & 1 & 4 & $5^{16}$ & 1 & 3 \\
\hline 1 & 2 & 3 & $2^{16}$ & 1 & 6 & $3^{15}$ & 3 & 5 & $5^{15}$ & 1 & 5 \\
\hline 1 & 3 & 2 & $2^{16}$ & 1 & 7 & $3^{15}$ & 1 & 6 & $5^{15}$ & 1 & 6 \\
\hline 2 & 2 & 3 & $2^{18}$ & 1 & 4 & $3^{19}$ & 1 & 4 & $5^{18}$ & 1 & 4 \\
\hline 2 & 2 & 4 & $2^{20}$ & 1 & 4 & $3^{21}$ & 1 & 5 & $5^{20}$ & 1 & 4 \\
\hline 1 & 3 & 5 & $2^{22}$ & 2 & 7 & $3^{21}$ & 9 & 6 & $5^{21}$ & 5 & 6 \\
\hline 1 & 5 & 3 & $2^{22}$ & 2 & 9 & $3^{21}$ & 9 & 8 & $5^{21}$ & 5 & 8 \\
\hline 3 & 3 & 3 & $2^{24}$ & 1 & 3 & $3^{25}$ & 1 & 4 & $5^{24}$ & 1 & 3 \\
\hline 1 & 4 & 6 & $2^{26}$ & 4 & 8 & $3^{25}$ & 27 & 7 & $5^{25}$ & 25 & 7 \\
\hline 1 & 6 & 4 & $2^{26}$ & 4 & 10 & $3^{25}$ & 27 & 9 & $5^{25}$ & 25 & 9 \\
\hline 2 & 4 & 5 & $2^{28}$ & 1 & 5 & $3^{28}$ & 1 & 5 & $5^{28}$ & 1 & 5 \\
\hline 2 & 4 & 6 & $2^{30}$ & 1 & 5 & $3^{30}$ & 3 & 5 & $5^{30}$ & 1 & 5 \\
\hline
\end{tabular}

OSSERvaZione 6. Non è difficile dimostrare che se $p \neq 20$ se $p^{\alpha}, p^{\beta}$ e $p^{\gamma}$ sono tutti maggiori di 4 allora $R M\left(p^{\alpha}, p^{\beta}, p^{\gamma}\right)$ è un $p$-gruppo powerful nel senso della definizione data in [8]. Ne segue che se $M(r, s, t)$ è finito allora un suo $p$-sottogruppo di Sylow è un $p$-gruppo powerful per ogni numero primo dispari $p$ (si rammenti che $M(r, s, t)$ è supersolubile e si veda 5.4 .8 di [12]).

OSSERVAZIONE 7. Vi è un altro caso in cui $M(r, s, t)$ risulta finito. Si supponga infatti $r=-2$ e sia $s=2 s_{1}+1$ dispari; allora $\rho=(1+r)^{|s|}-1=-2$, $\sigma=(1+s)^{|t|}-1$ e $\tau=(1+t)^{2}-1$. Condizione necessaria e sufficiente affinché $M\left(-2,2 s_{1}+1, t\right)$ sia finito è che $\rho, \sigma$ e $\tau$ siano tutti diversi da 0 , il che accade se e solo se $t \notin\{-2,0\}$. In questo caso si può dimostrare che

$$
\left|M\left(-2,2 s_{1}+1, t\right)\right|=|\rho \sigma \tau| .
$$


Osservazione 8. Mentre questo lavoro era in fase di revisione è apparsa la pubblicazione [2] in cui vengono studiati i gruppi $G(a, b ; c, d ; e, f)$ definiti dalla presentazione

$$
\left\langle x, y, z \mid\left(x^{a}\right)^{y}=x^{b},\left(y^{c}\right)^{z}=y^{d},\left(z^{e}\right)^{x}=z^{f}\right\rangle ; \quad a, b, c, d, e, f \in Z \text {. }
$$

Si ha ovviamente $M(r, s, t)=G(1,1+r ; 1,1+s ; 1,1+t)$ e quindi i gruppi $G(a, b ; c, d ; e, f)$ costituiscono una generalizzazione di quelli considerati nel presente lavoro. Il Teorema 2 di [2] stabilisce che, se $(a, b)=$ $=(c, d)=(e, f)=1$ e se nessuna delle tre coppie di parametri è uguale a $( \pm 1, \pm 1)$, allora $G(a, b ; c, d ; e, f)$ ammette un quoziente universale $Q=Q(a, b ; c, d ; e, f)$ tra quelli in cui $x, y$ e $z$ hanno ordine finito, $Q$ è finito e risolubile e $Q^{\prime}$ è nilpotente di classe al più due.

Non è difficile vedere come, utilizzando con poche modifiche la dimostrazione del Teorema 1 , sia possibile affermare che risulta $Q^{\prime \prime} \leq Z(Q)$ (si veda l'Osservazione 2).

Si osservi anche che in [11] è dimostrato che, se $1 \leq a<b$, $1 \leq c<d$ e $(a, b)=(c, d)=1$, allora $G(a, b ; c, d ; 1, f)$ ha ordine finito (si veda anche il Lemma 6 di [2]). D'altro canto è facile dimostrare che $Q(n, n+1 ; n, n+1 ; n, n+1)=\{1\}$ per ogni $n \in Z$; tenendo conto di alcuni risultati ottenuti in [2] appare ragionevole formulare la seguente

Congettura. Il gruppo $G(n, n+1 ; n, n+1 ; n, n+1)$ risulta infinito per ogni $n \in \mathbb{Z} \backslash\{-2,-1,0,1\}$.

In [10] Neumann afferma che se $2 \leq a \leq|b|, 2 \leq c \leq|d|$ e $2 \leq e \leq|f|$ allora $G(a, b ; c, d ; e, f)$ è un gruppo infinito; purtroppo la dimostrazione da lui fornita non è corretta.

Ringraziamenti. L'autore esprime la sua gratitudine all'anonimo referee per l'attenta e puntigliosa lettura del testo, grazie alla quale alcuni errori e parecchie inesattezze hanno potuto essere eliminati dalla versione finale di questo lavoro.

\section{BIBLIOGRAFIA}

[1] M. A. Albar - A. A. Al-Shuaibi, On Mennicke groups of deficiency zero. II. Canad. Math. Bull., 34, no. 3 (1991), pp. 289-293.

[2] D. Allcock, Triangles of Baumslag-Solitar groups. arXiv:0808.0934v1 ([v1] Wed, 6 Aug 2008; http://arxiv.org/abs/0808.0934v1).

[3] D. Gorenstein, Finite groups. Second edition. Chelsea Publishing Co., New York, 1980. 
[4] G. Higman, A finitely generated infinite simple group. J. London Math. Soc. 26, (1951). 61-64.

[5] N. Itô. Über das Produkt von zwei abelschen Gruppen. Math. Z. 62 (1955), 400-401.

[6] E. Jabara, Gruppi fattorizzati da sottogruppi abeliani. Rend. Sem. Mat. Univ. Padova. [In corso di pubblicazione]

[7] D. L. Johnson, E. F. Robertson, Finite groups of deficiency zero. Homological group theory (Proc. Sympos., Durham, 1977), pp. 275-289, London Math. Soc. Lecture Note Ser., 36, Cambridge Univ. Press, Cambridge-New York, 1979.

[8] A. Lubotzky, A. Mann, Powerful p-groups. I. Finite groups. J. Algebra 105 (1987), no. 2, 484-505.

[9] J. Mennicke, Einige endliche Gruppen mit drei Erzeugenden und drei Relationen. Arch. Math. 10 (1959) 409-418.

[10] B. H. Neumann, Some group presentations. Canad. J. Math. 30 (1978), no. 4, 838-850.

[11] M. Post. Finite three-generator groups with zero deficiency. Comm. Algebra 6 (1978), no. 13, 1289-1296.

[12] D. J. S. Robinson, A course in the theory of groups. Graduate Texts in Mathematics, 80. Springer-Verlag, New York-Berlin, 1982.

[13] E. Schenkman, A factorization theorem for groups and Lie algebras. Proc. Amer. Math. Soc. 68 (1978), no. 2, 149-152.

Manoscritto pervenuto in redazione il 4 luglio 2008 